\title{
ALGEBRAS OF DIFFERENTIABLE FUNCTIONS
}

\author{
BY K. DE LEEUW ${ }^{1}$ AND H. MIRKIL ${ }^{2}$
}

Communicated by Walter Rudin, March 18, 1962

\section{Classification of certain spaces of continuously differentiable} functions of two variables. Denote by $C_{0}$ the space of all complexvalued continuous functions on the plane that are zero at infinity. Write $\|\cdot\|_{\infty}$ for the supremum norm on $C_{0}$. Denote by $D$ the dense subspace of $C_{0}$ consisting of infinitely differentiable functions with compact support.

Throughout we shall be concerned with differential operators of the form

$$
\sum a_{m, n} \frac{\partial^{m+n}}{\partial x^{m} \partial y^{n}}
$$

the $a_{m, n}$ are complex constants. For each set $Q$ of such operators, we define $C_{0}(Q)$ to be the space of all $f$ in $C_{0}$ having $A f$ in $C_{0}$ (in the sense of Laurent Schwartz) for all $A$ in $Q$. Equivalently, $C_{0}(Q)$ is the completion of $D$ under the seminorms

$$
f \rightarrow\|f\|_{\infty} \text { and } f \rightarrow\|A f\|_{\infty}
$$

$A$ in $a$.

Each $C_{0}(Q)$ so defined is a translation-invariant space of functions; those that are furthermore invariant under rotations of the plane will be called rotating spaces of differentiable functions.

Certain of these spaces are familiar, namely the spaces $C_{0}^{N}$ consisting of those functions in $C_{0}$ that have all derivatives of order $\leqq N$ in $C_{0}$, and the space $C_{0}^{\infty}$, which is $\bigcap_{N} C_{0}^{N}$. A rotating space of differentiable functions will be called proper if it is not one of the $C_{0}^{N}$ and not $C_{0}^{\infty}$. Here is the classification of such spaces.

We use the notation

$$
\frac{\partial}{\partial z}=\frac{1}{2}\left(\frac{\partial}{\partial x}-i \frac{\partial}{\partial y}\right) \text { and } \frac{\partial}{\partial \bar{z}}=\frac{1}{2}\left(\frac{\partial}{\partial x}+i \frac{\partial}{\partial y}\right) .
$$

Theorem 1.1. If $a$ is a proper subset of

$$
\left\{\partial^{m+n} / \partial z^{m} \partial \bar{z}^{n}: m+n=N\right\}
$$

1 This research is supported in part by the United States Air Force Office of Scientific Research under contract AF 49(638)-294.

2 This research is supported in part by N.S.F. Grant G14242 and by the United States Air Force Office of Scientific Research under contract AF 49(638)-812. 
for $N$ a positive integer, then $C_{0}(a)$ is a proper rotating space of differentiable functions between $C_{0}^{N}$ and $C_{0}^{N-1}$. If $Q_{1}$ and $a_{2}$ are distinct subsets of (1.2) then $C_{0}\left(Q_{1}\right)$ and $C_{0}\left(Q_{2}\right)$ are distinct. Each proper rotating space of differentiable functions is a $C_{0}(Q)$, where $Q$ is a proper subset of (1.2) for some $N$.

COROLlaRy 1.2. Every proper rotating space of differentiable functions is a Banach algebra under pointwise multiplication.

For instance, the six distinct proper rotating spaces of differentiable functions between $C_{0}^{1}$ and $C_{0}^{2}$ are

$$
\begin{aligned}
& C_{0}\left(\partial^{2} / \partial z^{2}\right), \\
& C_{0}\left(\partial^{2} / \partial \bar{z}^{2}\right), \\
& C_{0}\left(\partial^{2} / \partial z \partial \bar{z}\right)=C_{0}\left(\partial^{2} / \partial x^{2}+\partial^{2} / \partial y^{2}\right), \\
& C_{0}\left(\partial^{2} / \partial z^{2}, \partial^{2} / \partial \bar{z}^{2}\right)=C_{0}\left(\partial^{2} / \partial x^{2}-\partial^{2} / \partial y^{2}, \partial^{2} / \partial x \partial y\right), \\
& C_{0}\left(\partial^{2} / \partial z^{2}, \partial^{2} / \partial z \partial \bar{z}\right), \\
& C_{0}\left(\partial^{2} / \partial \bar{z}^{2}, \partial^{2} / \partial z \partial \bar{z}\right) .
\end{aligned}
$$

REMARK 1.3. Suppose instead of the supremum norm we take as basic norm $\|f\|_{p}=\left\{\int|f|^{p}\right\}^{1 / p}, 1<p<\infty$. Then there are no proper rotating spaces

$$
L_{p}(a)=\left\{f: f \in L_{p}, A f \in L_{p} \text { for all } A \in \mathbb{Q}\right\} .
$$

Indeed the only rotating spaces are the Sobolev spaces

$$
L_{p}^{N}=\left\{f: f \in L_{p},\left(\partial^{m+n} / \partial x^{m} \partial y^{n}\right) f \in L_{p}, m+n \leqq N\right\},
$$

analogs of the $C_{0}^{N}$; and $L_{p}^{\infty}$, which is identical with $C_{0}^{\infty}$.

In a sense, rotating spaces are the ones that have geometrical significance. From this point of view the correct definition in norm $\|\cdot\|_{\infty}$ of "Sobolev space" would include not only the $C_{0}^{N}$ but also the proper rotating spaces.

2. Spaces of continuously differentiable functions on Riemann surfaces. It is possible to define algebras of functions on Riemann surfaces corresponding to those described in Theorem 1.1 and to show that these algebras determine the conformal structure.

Let $U$ be an open subset of the plane, $C(U)$ the space of all complexvalued continuous functions on $U$. For $Q$ a set of differential operators of the form (1.1), we denote by $C(U, a)$ the subspace of $C(U)$ consisting of those $f$ in $C(U)$ with $A f$ in $C(U)$ (in the sense of Laurent Schwartz) for each $A$ in $Q$.

For a general set $Q$ of differential operators, the spaces $C(U, a)$ 
have no interesting invariance properties. However we have

Lemma 2.1. If $a$ is a subset of (1.2), the spaces $C(U, a)$ are invariant under conformal transformations.

This result allows the extension of the definition of the $C(U, a)$ to Riemann surfaces. If $R$ is a Riemann surface and $a$ a subset of (1.2), $C(R, Q)$ is defined to be the space of those functions on $R$ such that if $U=\{z:|z|<1\}$ and $\phi: U \rightarrow R$ is a coordinate disk, the composite function $f \circ \phi$ is in $C(U, a)$.

Each $C(R, a)$ is an algebra of functions on $R$, with multiplicative linear functionals corresponding to points of $R$, and with a natural complete locally convex topology. In this topology $C(R, a)$ is a Banach algebra if and only if $R$ is compact.

In three instances $C(R, a)$ can be described in terms of exterior differential operators defined globally on $R$.

$$
\begin{aligned}
C(R, \partial / \partial z) & =\{f: f \text { and } \partial f \text { continuous on } R\}, \\
C(R, \partial / \partial \bar{z}) & =\{f: f \text { and } \bar{\partial} f \text { continuous on } R\}, \\
C\left(R, \partial^{2} / \partial z \partial \bar{z}\right) & =\{f: f \text { and } \Delta f \text { continuous on } R\},
\end{aligned}
$$

where the operators $\partial, \bar{\partial}$ and $\Delta$, taking functions into differential forms, are defined in terms of any coordinate system by

$$
\partial f=\frac{\partial f}{\partial z} d z, \quad \bar{\partial} f=\frac{\partial f}{\partial \bar{z}} d \bar{z}, \quad \Delta f=\frac{1}{4} \frac{\partial^{2} f}{\partial z \partial \bar{z}} d z d \bar{z} .
$$

The following result states the extent to which the algebras $C(R, a)$ determine the conformal structure of $R$.

Theorem 2.2. Let $R_{1}$ and $R_{2}$ be connected Riemann surfaces and $a$ be a proper subset of (1.2). Each conformal equivalence of $R_{1}$ with $R_{2}$ induces an algebra isomorphism of $C\left(R_{1}, Q\right)$ with $C\left(R_{2}, a\right)$. If $a$ is symmetric (i.e., $\partial^{m+n} / \partial z^{m} \partial \bar{z}^{n}$ in $Q$ if $\partial^{m+n} / \partial z^{n} \partial \bar{z}^{m}$ in $Q$ ), an anticonformal equivalence of $R_{1}$ with $R_{2}$ also induces an algebra isomorphism of $C\left(R_{1}, a\right)$ with $C\left(R_{2}, \mathfrak{Q}\right)$. No other algebra isomorphisms of $C\left(R_{1}, a\right)$ with $C\left(R_{2}\right.$, Q $)$ are possible.

A similar result identifies all algebra homomorphisms of the $C(R, a)$.

3. Sup norm estimates. The work of the preceding sections is based on the existence and nonexistence of certain sup norm estimates for constant-coefficient differential operators. In this section we state 
these results, which may be of some independent interest. All these results remain valid for $n$ variables.

If $P$ is a polynomial,

$$
P(x, y)=\sum a_{m, n} x^{m} y^{n},
$$

we denote by $P^{N}$ its homogeneous part of degree $N$,

$$
P^{N}(x, y)=\sum_{m+n=N} a_{m, n} x^{m} y^{n},
$$

and by $\hat{P}$ its Fourier transform, the differential operator

$$
\sum(-i)^{m+n} a_{m, n} \frac{\partial^{m+n}}{\partial x^{m} \partial y^{n}} .
$$

An operator $\hat{P}$ of order $N$ is called elliptic if $P^{N}(x, y) \neq 0$ for $(x, y)$ $\neq(0,0)$.

Theorem 3.1. Let $Q, P_{1}, \cdots, P_{r}$ be polynomials of degree $N$ or less. Then the following are equivalent:

(1) There is a constant $K$ so that

$$
\|\hat{Q} f\|_{\infty} \leqq K\left(\left\|\hat{P}_{1} f\right\|_{\infty}+\cdots+\left\|\hat{P}_{r}\right\|_{\infty}\right)
$$

for all $f$ in $D$.

(2) There are finite measures $\mu_{1}, \cdots, \mu_{r}$ in the plane whose FourierStieltjes transforms $\hat{\mu}_{1}, \cdots, \hat{\mu}_{r}$ satisfy

$$
Q=P_{1} \hat{\mu}_{1}+\cdots+P_{r} \hat{\mu}_{r}
$$

If (1) and (2) hold, it is possible to find constants $c_{1}, \cdots, c_{r}$ so that

$$
Q^{N}=c_{1} P_{1}^{N}+\cdots+c_{r} P_{r}^{N} .
$$

Theorem 3.2. Let $\hat{P}$ be elliptic and of order $N \geqq 2$. Then for each $\hat{Q}$ of order strictly less than $N$ there is a constant $K$ so that

for all $f$ in $D$.

$$
\|\hat{Q} f\|_{\infty} \leqq K\left(\|\hat{P} f\|_{\infty}+\|f\|_{\infty}\right)
$$

CoROLlaRy 3.3. $\partial^{m+n} / \partial z^{m} \partial \bar{z}^{n}$ is elliptic. Hence, if $p+q<m+n$, there is a constant $K$ so that

$$
\left\|\left(\partial^{p+q} / \partial x^{p} \partial y^{q}\right) f\right\|_{\infty} \leqq K\left(\left\|\left(\partial^{m+n} / \partial z^{m} \partial \bar{z}^{n}\right) f\right\|_{\infty}+\|f\|_{\infty}\right)
$$

for all $f$ in $D$.

REMARK 3.4. The situation for estimates in $\|\cdot\|_{p}, 1<p<\infty$, is quite different, which is the reason for the phenomenon mentioned in Re- 
mark 1.3. To be precise, if $\hat{P}$ is elliptic of order $N, \hat{Q}$ arbitrary of order $\leqq N$, it is known that there is an estimate of the form

$$
\|\hat{Q} f\|_{p} \leqq K\left(\|\hat{P} f\|_{p}+\|f\|_{p}\right) \quad f \in D,
$$

and the existence of these estimates is characteristic of ellipticity. It is natural to ask whether the existence of sup norm estimates like those in Theorem 3.2 (where $\hat{Q}$ has strictly lower order) also characterizes ellipticity. In the plane the answer is no, but in higher dimensions yes.

STANFord UNIVERSITy AND

Dartmouth College 